Archived version from NCDOCKS Institutional Repository http://libres.uncg.edu/ir/asu/

\title{
Appalachľan
}

B O O NE, NORTH CAROLINA

\section{Predicting Ideal Body Mass Index: What Does Clothing Size Have To Do With It?}

\author{
By: Amy T. Galloway, Rose Mary Webb, Denise M. Martz \& Anna B. Petroff
}

\begin{abstract}
This study examined demographic and anthropometric variables as predictors of ideal body mass index (BMI) from cross-sectional, archival, self-reported data from the Psychology of Size Survey of 4014 U.S. residents collected in 2007. As hypothesized, ideal BMI can be predicted by a

within gender hierarchical multiple regression analysis with the predictor variables of age, number of clothing sizes from ideal size, and current BMI; these variables account for $54.1 \%$ of variance in women's ideal BMI and 65.5\% of variance in men's ideal BMI. Findings also demonstrated a logarithmic relationship between current BMI and ideal BMI, with increasing variance in ideal BMI for individuals with high current BMIs. These findings evidence the strong role of current body characteristics in the formation of ideals. Understanding how individuals conceptualize body ideals can inform researchers and practitioners alike, as this evidence has important implications related to both medical and psychological health.
\end{abstract}

Amy T. Galloway, Rose Mary Webb, Denise M. Martz \& Anna B. Petroff (2011) "Predicting Ideal Body Mass Index: What Does Clothing Size Have To Do With It?" Body Image \#8 pp:126-134 [DOI 10.1016/2011.02.003] Version of Record Available From (www.researchgate.net) 


\section{Introduction}

Currently in the United States (U.S.), record high overweight and obesity rates distance many individuals from cultural ideals of attractiveness, limit quality of life, and endanger overall health (Flegal, Carroll, Ogden, \& Curtin, 2010; Grabe, Ward, \& Hyde, 2008; Hassan, Joshi, Madhavan, \& Amonkar, 2003; Mokdad et al., 2003). Despite this epidemic, the mass media, possibly the strongest mode of communication of sociocultural standards, portrays attractive men as muscular and trim and beautiful women as slim and willowy, standards that have grown more normative in recent decades (Frederick, Fessler, \& Haselton, 2005; Heinberg, 1996; Leit, Pope, \& Gray, 2001; Mazur, 1986; Morry \& Staska, 2001; Spitzer, Henderson, \& Zivian, 1999; Sypeck, Gray, \& Ahrens, 2004). Although media images imply specific body ideals for men and women, individuals vary in the degree to which they adopt them as their personal ideals (Thompson \& Heinberg, 1999). Given the contrast between a mostly overweight society and very thin (for women) or muscular (for men) cultural ideals, it is clear that the personal ideals of men and women are determined by more than just cultural ideals. But what other variables might influence individuals' personal ideals?

Body image has been implicated as one such influence. Body image dissatisfaction, negative thoughts and feelings about one's current weight and shape, underpins all manner of negative health, behavioral, and affective problems for both men and women. Similar to how women's body dissatisfaction manifests in unhealthy behaviors and thought patterns (Levine \& Piran, 2004; Stice \& Shaw, 2004), men with muscle dissatisfaction are more likely to experience depression, disordered eating patterns, lower self-esteem (Olivardia, Pope, Borowiecki, \& Cohane, 2004), and anabolic steroid use (Grogan, Evans, Wright, \& Hunter, 2006; Pope, Phillips, \& Olivardia, 2000).

Measuring body dissatisfaction across genders, however, has proven to be complex, in part because men and women have divergent body goals that correspond to the different cultural ideals specific to each gender (Fallon \& Rozin, 1985; Olivardia et al., 2004; Pope et al., 2000). Men tend to value physical attractiveness and strength, whereas women place more value in sexual attractiveness and controlled weight (Feingold \& Mazzella, 1998). Rusticus and Hubley (2006) assessed the ability of the Multidimensional BodySelf Relations Questionnaire (MBRSQ), a frequently used body image assessment test given to both men and women, to make comparisons across gender, and found that body image is indeed a very different construct for men and women, lending support to previous findings. For example, intercorrelations among desired body characteristics are stronger for men than women, suggesting that more diffuse factors influence women's body image (Franzoi \& Shields, 1984; Kashubeck-West, Mintz, \& Weigold, 2005).

Furthermore, a recent functional magnetic imaging (fMRI) study found that women without clinically significant body image concerns were more likely than men to engage in serious selfevaluation when viewing overweight body type than when viewing 
thin models, whereas men did not engage in significant selfreflection in either condition. This finding suggests that the link between body fat and personal worth is stronger for women than men, even in women without body dissatisfaction (Owens, Allen, \& Spangler, 2010). Although that study could not address men's body dissatisfaction directly because it did not examine muscularity, the findings align with the theory that women have a deep-seated fear of becoming fat, which may impact weight-related body image dissatisfaction differently. Other previous research indicates that men are less likely to be aware they are overweight, are content with a heavier weight, and view lighter weight for men as unhealthy, all of which put them more at risk for slipping into overweight (Gregory, Blanck, Gillespie, Maynard, \& Serdula, 2008; McCreary, 2002; McCreary \& Sadava, 2001).

Because of the limitations and lingering questions regarding body image, the current study uses a novel method of assessing body image: relative clothing size, which is construed as a discrepancy score between one's current size and one's ideal size. Relative size corresponds precisely and consistently with National Institutes of Health (NIH) categories of overweight separately for women and men (Martz, Curtin, Williams, Burgess, \& Maphis, 2009), which suggests that the measure provides meaningful descriptions of body size and shape. Also, because people may think in terms of "goal size" rather than ideal weight, relative clothing size may be a practical measure of body image dissatisfaction.

It is important to note that the discrepancy between current and ideal sizes may be due not only to body image dissatisfaction but also health concerns. Including relative size allows for interpretations about health because it is known that waist circumference estimates pathogenic adipose tissue beyond body mass index (BMI) alone (Fox et al., 2007; Janssen, Heymsfield, Allison, Kotler, \& Ross, 2002). For those who are obese, health may be the more salient concern than issues of appearance (Munoz et al., 2007; Putterman \& Linden, 2004; Reas, Masheb, \& Grilo, 2004). However, the perception of a "healthy size" may present differently for men and women given different body ideals (Flegal, Carroll, Ogden, \& Johnson, 2002; McCreary \& Sadava, 2001; Neighbors \& Sobal, 2007). For this reason, in this initial study of the utility of relative size in predicting personal ideals, we will conservatively test its predictive power by examining it separately by gender.

In addition to the complexities of how body image contributes to one's personal ideal, a person's current weight status is arguably a salient variable in how individuals imagine their ideal body. However, even this straightforward variable does not operate consistently for men and women. For example, past research indicates that men are less likely than women to be aware they are overweight, are content with a heavier weight, and view lighter weight for men as unhealthy, all of which put them more at risk for slipping into overweight (Gregory et al., 2008; McCreary, 2002; McCreary \& Sadava, 2001). Moreover, studies in undergraduate female populations suggest that heavier young women may have larger personal body ideals compared to cultural body ideals, possibly to prevent internalizing shame of being larger than the cultural standard and to resist ideals that may spur unhealthy weight loss methods in order to achieve that standard (Bessenoff \& Snow, 2006).

Consistent with the theory that ideal weight is relative to current weight, Neighbors and Sobal (2007) found strong correlations between current BMI and ideal BMI for 310 undergraduate men (.74) and women (.86). In samples of which $44 \%$ of men and $11 \%$ of women were classified as overweight, $59 \%$ of overweight men and $48 \%$ of overweight women reported an ideal weight that was still in the overweight range, according to the World Health Organization's (WHO, 1998) BMI categories which correspond to those used in the U.S. (NIH, 1998). These results are intriguing, yet it is unknown if there is a similar pattern for non-undergraduate populations across the U.S., which have a substantially higher proportion of overweight and obese individuals (Flegal et al., 2002). Therefore, the current study will also use current weight status to predict personal ideals.

Finally, age has been examined as a potential predictor of weight status and body image. Men and women are both likely to gain weight as they age, but women are more likely than men to experience substantial weight gain (Keel, Baxter, Heatherton, \& Joiner, 2007; Kuczmarski, 1992). Moreover, men and women's body image seems to have different significance for individuals at different ages (Rusticus \& Hubley, 2006). Despite the likelihood that women gain more weight than men, body dissatisfaction decreases for women and increases for men (Heatherton, Mahamedi, Striepe, Field, \& Keel, 1997; Kaminski \& Hayslip, 2006; Keel et al., 2007; Tiggemann \& Stevens, 1999; Tunaley, Walsh, \& Nicolson, 1999). However, our understanding of the relationship between age and body image is not yet definitive. For example, a study of 52,677 heterosexual men and women found that age was largely unrelated to body satisfaction (Frederick, Peplau, \& Lever, 2006). Because studies report contradictory evidence on this issue, the current investigation will attempt to further inform the debate by including age in the prediction of personal ideals.

Despite considerable research identifying gender differences in body dissatisfaction at different ages and weights, how these differences manifest in individual body ideals is largely unknown. The current investigation aims to elucidate that relationship. However, the first step in understanding the relationship is to see if the structure amongst those variables replicates across genders. Therefore, the current study examines the demographic variable of age, the anthropometric variable of current body mass, and the novel body image variable of relative clothing size, separately by gender, to predict ideal body mass index.

Unencumbered by the college convenience samples that limit generalization of findings in many body image studies, this study uses participant-reported data from a national, cross-sectional pool of 4014 online respondents obtained in 2007 to assess to what extent these variables predict ideal body mass index. We hypothesized that participants' current BMIs would be larger than their ideal BMIs, and we expected women to manifest greater discrepancies between current and ideal BMI than men. Moreover, we predicted that individuals would desire different ideal BMIs, specifically that those with higher current BMIs would have higher ideal BMIs. Further, we expected that, within gender, body ideals could be predicted by age, current BMI, and relative size. Understanding the predictors of ideal BMI can aid in the creation of more accurate body satisfaction measures, further understanding of how people conceptualize their personal body ideals, inform health outcomes relative to ideals, and perhaps aid in the development of more informed treatments for body image disorders and obesity.

\section{Method}

\section{Participants}

Participants included 4,014 U.S. citizens (2,007 men, 2,007 women), who were at least 18 years of age $(M=45.43$ years, $S D=15.68$ years). All information was self-reported. Participants with anatomically impossible combinations of height, weight, and waist size and those who misreported desired weight loss (e.g., desire to weigh zero pounds) were deemed outliers. After outliers and those with incomplete responses were removed, 3,906 participants (1,958 women, $50.1 \% ; 1,948$ men, $49.9 \%)$ remained in the final data set.

The majority of participants were Caucasian with some representation from other ethnic backgrounds. To compare our representativeness to the U.S. population, the first number pre- 
sented after race is the percentage of our participants, whereas the second number is the percentage reported by the U.S. Census Bureau (2001): Caucasian (83.7\%; 75.1\%), African American (5.7\%; 12.3\%), Asian/Pacific Islander (4.9\%; 3.6\%), Hispanic/Chicano/Latino (3.5\%; $12.5 \%$; U.S. Census Bureau notes that Hispanics may be of more than one race hence their percentages add up to over $100 \%)$, multiethnic (1.3\%; 7.9\%), and Native American/Indian (0.8\%; 0.9\%). While Caucasian individuals were well represented, some minority groups were underrepresented.

Considering the median household income was $\$ 52,029$ according to the U.S. Census Bureau (2009), our sample was close in representation yet indicating some overrepresentation in households earning between $\$ 35 \mathrm{~K}$ and $\$ 75 \mathrm{~K}$. Numbers in parentheses represent the percentage of households falling into each range in the last published census. Participants reported their total family income per year with $28.9 \%$ (36.0\%) at less than $\$ 35,000,21.1 \%$ (14.1\%) between $\$ 35,000$ and $\$ 49,999,22.4 \%$ (18.1\%) between $\$ 50,000$ and $\$ 74,999,11.8 \%$ (11.5\%) between $\$ 75,000$ and $\$ 99,999$, $7.8 \%$ (11.9\%) between $\$ 100,000$ and $\$ 149,999$, 3.6\% (0.1\%) at or above $\$ 150,000$, and $4.4 \%$ chose not to disclose this information. As indicated, there was some variance in the how reflective these participants were of the general population. Participant employment status was as follows: $45.9 \%$ indicated full-time employment, $11.1 \%$ indicated part-time employment, $5.1 \%$ were students, $10.2 \%$ identified as full-time homemakers, $20.3 \%$ reported they were retired, and $7.5 \%$ were currently not employed. Participant education ranged from 8 th grade or less $(0.3 \%)$, some high school $(2.2 \%)$, high school graduate (21.7\%), some college (36.8\%), college graduate (26.7\%), and post-graduate study (12.3\%).

\section{Materials}

\section{Psychology of size survey}

Designed by Martz and The Segmentation Company in 2007, the survey contained over 130 items intended for use with male and female participants. The survey included demographic information, attitudes towards current body image, physical measures of body size, ideal body size, and perceived pressure to achieve an ideal size. Question order within each topic heading was randomized, and some questions were presented on a conditional basis (e.g., only participants who reported having children were asked how many in a follow-up question). The following key variables originate from this survey.

\section{Current body mass index}

As our anthropometric measure, body mass index (BMI) was calculated as weight in kilograms divided by height in meters squared. Height in inches and weight in pounds, which were the final two questions on the survey, composed the values for calculating "current BMI" (metric). These values were self-reported, which have demonstrated a strong positive correlation (between .90 and .95) with technician-measured weight (McAdams, Van Dam, \& $\mathrm{Hu}, 2007)$. It is important to note that some systematic reporting biases in participant-reported weights exist though: Women tend to under-report their weight, men may under- or over-report weight, and heavier individuals under-report their weight (Cash, Grant, Shovlin, \& Lewis, 1992; McCreary, 2002; Shapiro \& Anderson, 2003). However, although this variance in accuracy across gender and weight is well documented, these researchers and others have characterized participant reporting of weight as fairly accurate. BMI is a widely used measure known to correlate with direct measures of body fat (Garrow \& Webster, 1985; Mei, Grummer-Strawn, Pietrobelli, Goulding, Goran, \& Dietz, 2002; Revicki \& Israel, 1986).
The participants were compared by BMI according to NIH's (1998) medically based-classification system.

\section{Relative size}

To consider participant distance from ideal size without introducing any statistical dependence with the ideal BMI, the authors included survey items regarding "relative size" as a novel measure of body image, calculated as the number of clothing sizes between participant-reported current and ideal clothing sizes. This measure used dress sizes for women and pants/waist sizes for men, and included allotment for the even-numbered women's sizing and the numerically sequential units (inches) in men's sizing. Relative size allowed us to group participants as under ideal size, at ideal size, and up to 5 or more sizes above ideal size (for 7 total intervals).

\section{Age}

Age was calculated as years aged since time of birth to the time of the survey in May 2007.

\section{Ideal BMI}

Ideal BMI is used to refer to each participant's ideal body size. This question was not asked directly. The authors used the data regarding desired weight change in pounds, if any, to reach ideal BMI and applied it to the formula for current BMI. Ideal BMI was calculated as: (total body weight in kilograms + or - the desired weight change in kilograms)/height in meters squared. Hence, what we have used as ideal BMI in this study was a function of participants' current height and weight and their desired change in body weight.

\section{Procedures}

The data for this study were obtained from a large-scale crosssectional survey administered by The Segmentation Company, a division of Yankelovich, between May 11 and May 18, 2007. The survey participants, preexisting members of a general online research panel called MyView Surveys, received e-mail invitations to participate in the study, entitled "Health and Wellness Survey." Due to the voluntary nature of the online survey, consent was inherent and participants received $\$ 1$ Pay-Pal ${ }^{\mathrm{TM}}$ vouchers. SlimFast ${ }^{\mathrm{TM}}$ sponsored the project but was not identified in the survey. Institutional Review Board approval was received on May 8, 2008.

\section{Statistical analyses}

Statistical analyses were computed with SPSS ${ }^{\circledR} 17.0$ (used for main analyses) and Matlab ${ }^{\circledR}$ \& Simulink ${ }^{\circledR}$ Student Version R2009a (used for specialty analyses such as those involving bootstrapping and for graphs). A validity check on the novel measure of body image, relative clothing size, was completed first, using it and gender to predict current BMI in a factorial ANOVA. Gender comparisons were then made using independent $t$-tests. Bivariate relationships were examined with Pearson and Spearman correlations. Finally, three-step hierarchical regression analyses were used, by gender, to examine the utility of age, current BMI, and relative size in the prediction of participants' ideal BMI.

\section{Results}

\section{Validity check of relative size}

To test whether relative size (RS) increments are equivalent for men and women, despite the natural difference in how clothing 
Table 1

Categories of current body mass index (BMI) percentages for participants (NIH, 1998).

\begin{tabular}{|c|c|c|c|c|}
\hline \multirow[t]{2}{*}{ BMI category and range } & \multicolumn{2}{|c|}{ Percentage of women $(n=1,958)$} & \multicolumn{2}{|c|}{ Percentage of men $(n=1,948)$} \\
\hline & Current BMI & Ideal BMI & Current BMI & Ideal BMI \\
\hline Underweight, 14.5-18.49 & 2.6 & 4.9 & 1.8 & 1.6 \\
\hline Lower healthy, 18.5-20.99 & 8.9 & 23.0 & 5.4 & 7.5 \\
\hline Middle healthy, 21.0-22.99 & 10.6 & 27.4 & 10.5 & 17.8 \\
\hline Upper healthy, 23.0-24.99 & 12.6 & 22.3 & 14.2 & 25.6 \\
\hline Lower overweight, 25.0-27.49 & 16.9 & 13.4 & 21.5 & 27.2 \\
\hline Upper overweight, 27.5-29.99 & 11.3 & 4.6 & 14.3 & 11.9 \\
\hline Obese $30.0+$ & 37.1 & 4.0 & 32.3 & 8.0 \\
\hline
\end{tabular}

Note: $\mathrm{NIH}=$ National Institute of Health.

size is measured for the genders, a 2 (gender) by 7 (RS) ANOVA was run with current BMI as the dependent variable. Although there was a negligible BMI difference between the genders, $F(1$, $3892=29.47, p<.01, \eta_{p}^{2}<.01$, the key results showed that each increment in RS increase revealed a significant increase in BMI, $F(6$, 3892 ) $=892.58, p<.01, \eta_{P}^{2}<.58$ (all $p$ s for posthoc tests between each RS category <.01).

\section{Gender comparisons of size}

Fig. 1 illustrates how men and women's is current and ideal BMI related to relative size. The authors investigated the gender differences in current BMI, ideal BMI, and relative size standings. Table 1 presents the current BMI percentages for male and female participants compared by NIH categories. Both women and men had current BMI means in the "upper overweight" NIH BMI category. Similar to Ogden, Carroll, Curtin, McDowel, Tabak, and Flegal's (2006) findings from the 2003-2004 National Health and Nutrition Examination Survey (NHANES) that approximately 65\% of adults were overweight or obese, $68.1 \%$ of male participants and $65.3 \%$ of female participants in the current study were overweight or obese, suggesting that participant BMIs were similar to the overall population. Given this information and census data, the participants for this study appear to be at least fairly representative of the U.S. population, certainly more so than common studies on body image using male and female college students.

Women had a lower mean ideal BMI than men $F(1$, $3904=338.35, p<.001, \eta_{p}^{2}=.08$. Women's mean ideal BMI was in the "upper healthy" category $(M=25.11, S D=3.63)$, whereas the men's mean ideal BMI was in the "lower overweight" category $(M=23.06, S D=3.34)$

The majority of our participants desired to be a smaller clothing size ( $83.4 \%$ of women, $70.3 \%$ of men). On average, women were two clothing sizes over their ideal size $(M=2.00, S D=1.53)$, and men were between one and two clothing sizes over their ideal size $(M=1.41, S D=1.15)$. Table 2 presents women and men's current BMI and ideal BMI means by relative size and provides the NIH BMI ranges. Men were more likely than women to have an overweight ideal BMI. Interestingly, men who described themselves as two sizes overweight were obese by NIH classification, whereas women described themselves as three sizes overweight before they were considered obese by NIH classification, suggesting that there is a gender difference in the perception of overweight status, although this finding may be an artifact of differing clothing metrics for men and women.

To further inform the body image concern that most women would want to be smaller but a discrepancy for men typically presents in a desire to be more muscular, we found that only $4.7 \%$ of men and $1.9 \%$ of women reported a desire to be a larger clothing size, whereas $25.0 \%$ of men and $14.7 \%$ of women were at their ideal size. The emphases of these descriptive analyses is that the majority of our sample, regardless of gender, desired to have a smaller clothing size, thereby reducing our concern about conceptual and measurement issues comparing body image dissatisfaction between women and men.

\section{Relationship between current and ideal BMI}

Supporting our hypotheses, individuals at higher current BMIs have higher ideal BMIs, as indicated by both rank-order correlations and logarithmic functions. Spearman's rho rank-order correlations indicate that the correlation between current BMI and ideal BMI for women is $r_{\mathrm{s}}=.69, p<.001$ and men is $r_{\mathrm{S}}=.77, p<.001$ indicating that an individual's standing on current BMI corresponded to his or her ideal BMI (see Table 3).

While it is generally true that individuals at higher current BMIs have higher ideal BMIs, this relationship is limited by the restriction of range in ideal BMI; that is, there is a smaller range of healthy (ideal) BMI values and a wider range of actual BMI values. A logarithmic function can describe the naturally occurring sublinear relationship between current BMI and ideal BMI. The function has a steep slope at low to average current BMIs and a modest slope at high current BMIs. This logarithmic representation of the data is presented in Fig. 1, with each datum point in the graph representing the mean current BMI value of a bin of 25 similar individuals (for interpretable viewing). As shown, there is a sublinear relationship in which those with higher current BMIs have higher ideal

Table 2

Categories of current body mass index (BMI) means and ideal BMI means for women and men by relative size group.

\begin{tabular}{|c|c|c|c|c|c|c|c|c|c|c|}
\hline \multirow[t]{3}{*}{ Relative size } & \multicolumn{5}{|c|}{ Women } & \multicolumn{5}{|l|}{ Men } \\
\hline & \multirow[t]{2}{*}{$n$} & \multicolumn{2}{|c|}{ Current BMI } & \multicolumn{2}{|c|}{ Ideal BMI } & \multirow[t]{2}{*}{$n$} & \multicolumn{2}{|c|}{ Current BMI } & \multicolumn{2}{|c|}{ Ideal BMI } \\
\hline & & Mean & NIH category & Mean & NIH category & & Mean & NIH category & Mean & NIH category \\
\hline Smaller & 33 & 19.20 & Lower healthy & 21.40 & Middle healthy & 89 & 21.26 & Middle healthy & 23.99 & Upper healthy \\
\hline Current & 290 & 21.70 & Middle healthy & 21.73 & Middle healthy & 497 & 24.09 & Upper healthy & 24.09 & Upper healthy \\
\hline+1 & 495 & 24.99 & Upper healthy & 22.87 & Middle healthy & 598 & 26.86 & Lower overweight & 24.67 & Upper healthy \\
\hline+2 & 507 & 28.71 & Upper overweight & 23.23 & Upper healthy & 393 & 30.29 & Obese & 25.86 & Lower overweight \\
\hline+3 & 289 & 32.66 & Obese & 24.14 & Upper healthy & 160 & 32.88 & Obese & 26.30 & Lower overweight \\
\hline+4 & 144 & 35.33 & Obese & 23.97 & Upper healthy & 89 & 35.59 & Obese & 26.95 & Lower overweight \\
\hline$\geq 5$ & 200 & 42.70 & Obese & 24.59 & Upper healthy & 122 & 42.10 & Obese & 26.97 & Lower overweight \\
\hline
\end{tabular}

Note: $\mathrm{NIH}=$ National Institute of Health. 
Table 3

Correlations among relative size, current body mass index (BMI), ideal BMI, and age.

\begin{tabular}{lllll}
\hline Measure & 1 & 2 & 3 & 4 \\
\hline Women $(n=1,958)$ & & & & \\
1. Relative size & - & $.78^{* *}$ & $.28^{* *}$ & $-.06^{* *}$ \\
2. Current BMI & & - & $.69^{* *}$ & $.07^{* *}$ \\
3. Ideal BMI & & & - & $.13^{* *}$ \\
4. Age & & & - \\
Men $(n=1,948)$ & $.73^{* *}$ & $.26^{* *}$ & $.19^{* *}$ \\
1. Relative size & - & - & $.77^{* *}$ & $.18^{*}$ \\
2. Current BMI & & & - & $.12^{* *}$ \\
3. Ideal BMI & & & & -
\end{tabular}

Values in table represent Spearman's rho rank-order correlations between current BMI and ideal BMI; all other values represent Pearson's correlations.

${ }^{*} p<.01$.

$p<.001$.

BMIs; however, the degree of positive upwards slope decreases when current BMI values reach heavy overweight and obese levels. Interestingly, the point at which the slope decreases is approximately where the current BMI is in the NIH's categories for "upper overweight" and "obesity." This finding illustrates that participants with current BMIs in the obese range $(B M I \geq 30)$ seldom have ideal BMI values that are also in that range, suggesting strong health and appearance concerns in choosing an ideal BMI.

Additionally, when current BMI is grouped into bins of 25 data points and the mean and variance of each bin are calculated, the variance of values around mean ideal BMIs increases with current BMI (presented in Fig. 2). The binned current BMI rank-order correlations of variance of ideal BMI with current BMI for women is $.93(95 \% \mathrm{CI}: .90, .97)$ and for men is $.90(95 \% \mathrm{CI}: .84, .95)$, illustrating that those with a higher current BMI have greater variance in ideal BMI. This finding may help to explain the wide confidence intervals around the parameter estimates in the equations relating current and ideal BMIs.

Bivariate associations among current and ideal BMI, relative size, and age

The strength of association among current BMI, ideal BMI, relative size, and age separately for men and women is described in Table 3. The strong positive correlation between relative size and current BMI lends evidence that our measures reflect similar

Current BMI, Ideal BMI, and Relative Size

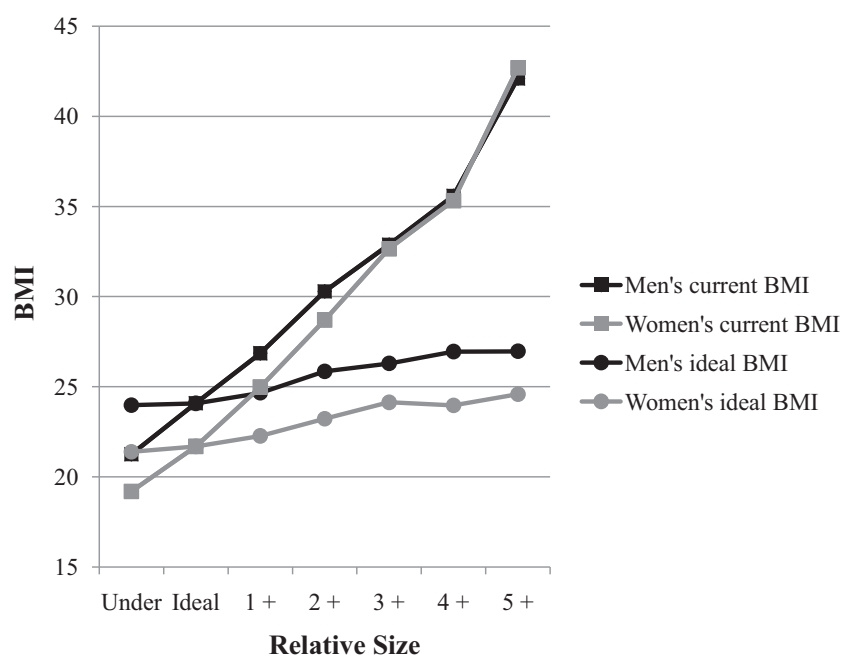

Fig. 1. Gender differences in current BMI and ideal BMI by relative size group.

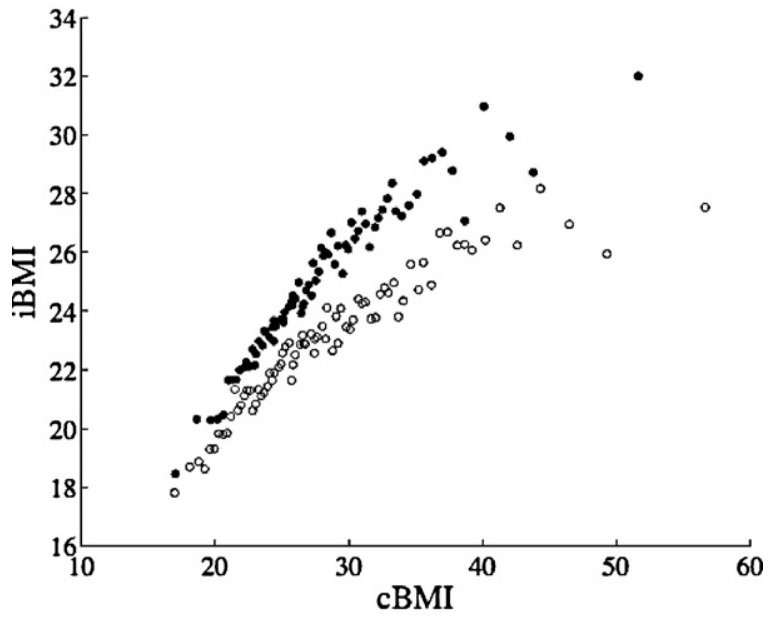

Fig. 2. Scatterplot between current BMI (cBMI) and ideal BMI (iBMI) for men (solid circle) and women (ring). Each data point represents a bin of 25 individuals. These data points follow a logarithmic function of the equation $\mathrm{iBMI}=[\mathrm{m} \log (\mathrm{cBMI})]+\mathrm{b}$ where $m=30.249$ (95\% CI: 27.454, 33.043) and $b=-18.602(95 \% \mathrm{CI}:-22.681$ $-14.523)$ for men, and $m=21.410$ (95\% CI: $18.819,24.001)$ and $b=-8.564(95 \% \mathrm{CI}$ : $-12.344,-4.785)$ for women.

aspects of discrepancy scores (but see also validity examination above). Contrary to findings reviewed above, the positive correlation between age and current BMI is slight for both men and women, as is the positive correlation between age and ideal BMI (Fig. 3).

\section{Age, relative size, and current BMI as predictors of ideal BMI}

Hierarchical regression analysis was used to predict ideal BMI for each gender using the following predictors: age (Step 1 ), relative size (Step 2), and current BMI (Step 3). These findings are presented in Table 4. For women, the first step in the regression revealed that age was a small but significant predictor of ideal BMI, $\Delta R^{2}=.01$, $p<.001$. Entering both age and distance from ideal clothing size, as measured in terms of relative size, was a more notable predictor of the ideal BMI, $\Delta R^{2}=.08, p<.001$. Entering age, relative size, and current $\mathrm{BMI}$ into the model resulted in the greatest ability to predict ideal BMI $\left(\Delta R^{2}=.44, p<.001\right)$. Although age was no longer a significant predictor of ideal BMI in the final model, relative size was. In this configuration, findings indicate that current BMI accounts

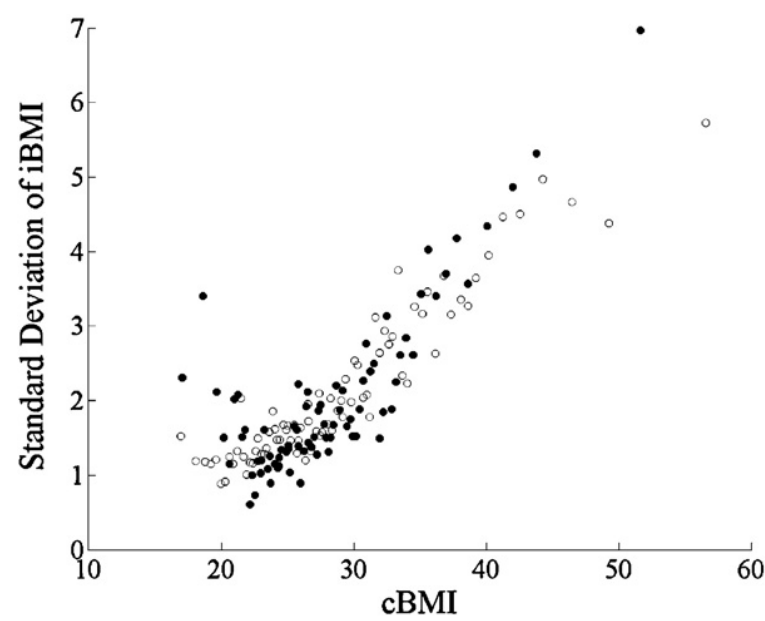

Fig. 3. Scatterplot between current BMI (cBMI) and standard deviations of ideal BMI (iBMI) for men (solid circle) and women (ring). This figure depicts greater variance in ideal BMI for those with higher current BMI. 
Table 4

Hierarchical regression analysis for women and men.

\begin{tabular}{|c|c|c|c|c|c|c|c|c|}
\hline \multirow[t]{2}{*}{ Variable } & \multicolumn{4}{|c|}{ Women $(n=1,958)$} & \multicolumn{4}{|c|}{ Men $(n=1,948)$} \\
\hline & $B$ & Adj. $R^{2}$ & $\beta$ & $\Delta R^{2}$ & $B$ & Adj. $R^{2}$ & $\beta$ & $\Delta R^{2}$ \\
\hline \multicolumn{9}{|l|}{ Step 1} \\
\hline Constant & 21.77 & & & & 23.87 & & & \\
\hline Age & 0.03 & .02 & $0.13^{* *}$ & .01 & 0.03 & .01 & $0.12^{* *}$ & .01 \\
\hline \multicolumn{9}{|l|}{ Step 2} \\
\hline Constant & 19.08 & & & & 22.29 & & & \\
\hline Age & 0.03 & & $0.15^{* *}$ & & 0.18 & & $0.07^{*}$ & \\
\hline Relative size & 0.63 & .10 & $0.29^{* *}$ & .08 & 0.61 & .07 & $0.25^{* *}$ & .06 \\
\hline \multicolumn{9}{|l|}{ Step 3} \\
\hline Constant & 14.20 & & & & 11.99 & & & \\
\hline Age & 0.01 & & 0.02 & & 0.01 & & 0.03 & \\
\hline Relative size & -1.22 & & $-0.56^{* *}$ & & -1.41 & & $-0.58^{* *}$ & \\
\hline Current BMI & 0.46 & .54 & $1.08^{* *}$ & .44 & 0.62 & .65 & $1.13^{* *}$ & .58 \\
\hline Total & & & & .54 & & & & .65 \\
\hline
\end{tabular}

$\mathrm{BMI}=$ body mass index.

${ }^{*} p<.01$.

** $p<.001$.

for an additional $44 \%$ of the variance in ideal BMI, making it by far the strongest of the three variables. While it is possible to use logarithmically transformed data for current BMI in this analysis, the results changed very little. Therefore authors opted to use and report the more directly interpretable linear results. Overall, this model explained a total of $54.1 \%$ of the variance in women's ideal BMI, with individuals older, further from their ideal clothing size, and at higher current BMI having higher ideal BMI.

For men, this hierarchical regression analysis revealed the following findings, which are also presented in Table 4. The factor of age indicated a slight ability to predict ideal BMI, $R^{2}=.01, p<.001$. Secondly, age and relative size resulted in stronger prediction of ideal BMI, wherein relative size accounted for additional variance, $\Delta R^{2}=.06, p<.001$. Similar to the findings in the model for women, age, relative size, and current BMI result in the strongest prediction of ideal BMI, with current BMI as the greatest contributor, $\Delta R^{2}=.58$, $p<.001$. This combination also reduced age to nonsignificant status in the prediction of ideal BMI, but relative size remained significant. The findings of these analyses indicate that the predictors accounted for more variance in men's ideal BMI than they did for women's ideal BMI. Overall, the model for men accounted for $65.5 \%$ of the variance in ideal BMI, $11.4 \%$ more than that for women.

\section{Discussion}

In recent decades, the prevalence of overweight and obesity has changed markedly, and the Centers for Disease Control estimates that these issues now affect approximately $65 \%$ of US adults (Flegal et al., 2002). This trend may remain the same or increase in light of data indicating that only $3 \%$ of the adult population adheres to all of the following basic health behaviors: maintaining a healthy weight below $25 \mathrm{BMI}$, eating five servings of fruits and vegetables a day, not smoking, and consistently exercising at or above minimum guidelines (Reeves \& Rafferty, 2005). Given the obesity epidemic and the low adherence to behaviors that could prevent high body mass, it is important to assess how individuals view ideal body mass both because it may reflect issues in body image dissatisfaction and how individuals today choose weight loss goals for health and appearance reasons. The results from this study provide evidence that demographic, anthropometric, and the novel body image measure of relative size contribute to personal ideal BMIs.

Using cross-sectional, archival data taken in 2007 of 3,906 U.S. adults, this study explored the relationships among current BMI as an anthropometric measure, ideal weight (ideal BMI), and number of sizes from ideal clothing size as the body image construct. The common thread of the predictions was that the current demographic, anthropometric, and body image characteristics of individuals can be used to better understand the underlying structure of ideal BMI. We found two ways to describe this structure: as a systematic relationship between current BMI and ideal BMI, and by the body image discrepancy between participants' current and ideal real-world clothing sizes. This study is the first to examine the underlying structure among these variables and will help further research in health, psychology, and gender studies, among other areas. The national, cross-sectional participant group that approximated racial and income representation of the U.S. population supports the generalizability of these findings, particularly considering that many body image studies include only college students.

\section{Gender, ideal BMI, and relative size}

Findings indicate a gender difference in ideal BMI, with women's average in the NIH's upper healthy range and men's average in the lower overweight range. However, because men and women have different body ideals that may influence how they conceptualized their ideal weight (i.e., ideal muscularity affects weight and consequently BMI), there are several factors to consider when interpreting these findings. Because body dissatisfaction is relative to both body image concerns and health concerns, evidence from both ideal BMI and relative size measures clarifies these findings.

In keeping with body image gender differences, it is arguable that when answering these questions, women considered losing weight whereas men considered gaining muscle (Fallon \& Rozin, 1985; Olivardia et al., 2004; Pope et al., 2000); however, there is emerging evidence that men and women have dual drives for lean and muscular bodies, findings that lend support to using BMI in this study (Bordo, 2003; Kelley, Neufeld, \& Musher-Eizenman, 2010). To glean insight about ideals that are true of both men and women, we look to the relative size measure, which is similar to measures of waist circumference because both men and women's clothing account for the dimensions of the abdomen. Although clothing size is not an exact measurement of waist circumference, it is thought to be an adequate reference point because it is known to estimate the NIH categories of overweight (Martz et al., 2009; Sauer, 2010). These abdominal measures help us understand the contribution of health concerns in overall body dissatisfaction. Waist circumference is known to provide estimates of abdominal, nonabdominal, and visceral adipose tissue beyond BMI alone (Janssen et al., 2002). This fat, particularly visceral adipose tissue, is known to be a particularly pathogenic fat (Fox et al., 2007). Inclusion of this relative size measure allows a unique view into the relative amounts of 
body dissatisfaction at different degrees of overweight and/or lack of muscularity.

As previously described, several studies found the drive for muscularity is important in determining men's body satisfaction (e.g., Thompson \& Cafri, 2007), which was not assessed in the current study's participants. However, we propose that clothing size may account for these mutual goals across the genders since a smaller size would indicate a smaller dress size for women, but an increase in muscularity and a decrease in weight for an overweight man would both likely contribute to a smaller waist size. Future studies are needed to illuminate how desired bodily changes for women and men correspond with both idealized weights (BMI) and idealized clothing sizes.

While more research is needed, this study found salient evidence regarding the degree to which men and women report ideal BMIs that are consistent with previously documented genderspecific patterns of body satisfaction. Because many of the women would need to lose weight to be in the upper healthy range, women's reported ideals are consistent with idealizing controlled weight, but because it is a heavier average than that espoused by media images it appears to reflect one of a number of possibilities. Only $5 \%$ of female participants desired an underweight BMI $(<18.5)$, similar to that portrayed in media images. Why there are so few women reporting an ultra-low BMI given the media pressures is perhaps related to the fact that the survey items assessed weight concerns but not characteristics such as height or bone structure, qualities that contribute to the supermodel-like appearance with corresponding ultra-low BMI. Another possible reason why so few women listed ultra-low ideal BMIs is that individuals vary in their internalization of media pressures (see Yamamiya, Cash, Melnyk, Posavac, \& Posavac, 2005). Despite these possibilities, the findings indicate that the strong majority of women do not desire a model-like figure, favoring goals that are healthy regardless of bone structure or body type. The average ideal BMI for men was in the lower overweight category, which is consistent with findings from other studies indicating that men are less likely to be aware they are overweight, are more likely to be content with a heavier weight, whether composed of muscle or fat, than women, and are more likely to view lighter weight for men as unhealthy (Gregory et al., 2008; McCreary \& Sadava, 2001). This finding may also reflect men's desire to achieve a muscular ideal. Another possible explanation for both men and women is that higher weights may be becoming normative, that is, a higher ideal may be both more acceptable and achievable.

\section{Predicting ideal BMI}

In support of our hypotheses, this study found two ways of describing the relationship between current body characteristics and ideal BMI: as a logarithm and with a regression analysis. The logarithmic relationship between current and ideal BMI describes ideals at current BMIs for men and women. For example, a female participant with a current BMI of 25 may have an ideal of 22, and a female participant with a current BMI of 31 may have an ideal of 26. However, there is more variance in ideal BMI at higher current $\mathrm{BMI}$ values, demonstrating that there may be more numerous influential factors for heavier people than for lean people. It could be that body image and health concerns operate differently relative to BMI, with weight loss desires at healthy current BMIs reflecting body image dissatisfaction and high current BMIs reflecting both body image dissatisfaction and health concerns (Munoz et al., 2007; Putterman \& Linden, 2004; Reas et al., 2004). Bessenoff and Snow (2006) suggested that heavier college women who report an ideal body size larger than the cultural ideal do so to cope with sociocultural pressures, to limit body shame, and to prevent eating disorders. Our findings suggest that this phenomenon may also be true of men but possibly with different patterns than women.

This study also revealed that age, relative size, and current BMI account for $54.1 \%$ of variance in women's ideal BMI and $65.5 \%$ of variance in men's ideal BMI. This finding aids in the understanding the role of anthropometric measures in reporting a body ideal. Relative size accounted for notable variance in ideal BMI, thus supporting this measure for estimating body dissatisfaction and health concerns, although more so for women than men. Current BMI was by far the strongest predictor, accounting for $44.1 \%$ of women's ideal BMI and 58.0\% of men's ideal BMI, even after controlling for age and relative size, supporting the hypothesis that there is a strong correspondence between current BMI and ideal BMI. Interestingly, age was the weakest predictor, adding only a very slight change in total variance before becoming nonsignificant when current BMI was added to the model. This suggests that whatever role age may play in influencing ideal BMI, it corresponds with and is overshadowed by the age-related trends in BMI gains across age. Contrary to previous findings, men were slightly heavier than women at more senior ages (Kuczmarski, 1992). The stability of the mean ideal BMIs for both men and women of differing ages may reflect that a healthy weight and satisfactory body image remain constant across age groups. However, the prevalence of eating disorders in young women that subsides towards middle age leaves the lingering question of the sociocultural influences on ideal BMI A longitudinal study that includes internalization of societal ideals as a predictor of ideal BMI may help to ascertain how internalization fluctuates with age despite consistent BMI. These reasons may be in part related to why this model accounted for $11.4 \%$ more variance for men than women, who may be subject to comparatively more diffuse factors that affect how women choose their ideal BMI. A future study could explore this idea by adding to the survey a measure of internalized acceptance of societal body physique ideals (Franzoi \& Shields, 1984). It is possible that this revised regression model could account for even more of the variance in ideal BMI.

Future research is needed to uncover explanations of these findings and may include one or a combination of the following three interpretations. One interpretation is that individuals at higher current BMIs may be more realistic when choosing ideal BMI because drastic weight change is difficult to achieve (Crawford, Jeffery, \& French, 2000; Serdula, Mokdad, Williamson, Galuska, Mendlein, \& Heath, 1999). A second interpretation is that personal history may constitute a personalized, ideal BMI reference point. For example, a woman's high school graduation weight or pre-pregnancy weight may represent her personal ideal. A complementary study would assess how ideal BMI changes with weight gain or loss especially if individuals keep an ideal in memory of a leaner period of their lives (e.g., prior to pregnancy). If one was happy with a past BMI but gains weight, is that pre-weight gain BMI the ideal BMI? If one achieves an ideal through diet and exercise, does a new, lower ideal BMI emerge shortly thereafter? Longitudinal studies are needed to explore these questions. A third interpretation is that there may be more weight complacency or attenuation for those at higher BMIs, especially considering the relative ease of weight gain for some individuals in our cultural environment that encourages sedentary behavior and supplies us with ample calorically dense foods, as compared to the extreme challenge that it takes for some overweight individuals to lose weight.

\section{Comparison to college population}

Neighbors and Sobal (2007) examined current and ideal BMIs in male and female college students using similar methodology for calculating the two variables of interest as the current study. When compared to the findings of the current study's examination of the general population, interesting differences emerge. While 
the relationship between current and ideal BMI for men is similar in (undergraduate $r=.74$ ) and national $\left(r=.71 ; r_{\mathrm{s}}=.77\right.$ ) populations, the link between current and ideal BMI for women is much stronger in the undergraduate subject pool $(r=.86)$ than the national subject pool $\left(r=.64 ; r_{\mathrm{s}}=.70\right)$. This comparison suggests that there may be an interesting difference in factors affecting the relationship between current and ideal BMI for women in these two populations. The findings from the current study present a possible explanation for this difference: Because overweight and obesity rates are much lower for undergraduate populations, there is less variance in the ideal BMI because individuals, on average, are closer to their goal weight, thus increasing the correlation between current and ideal BMI. This is likely a function of the undergraduates being less overweight and thus closer to their ideals naturally, whereas the national population reflected U.S. obesity rates indicating that more individuals were further from a normal weight ideal. This cursory comparison yields a possible direction for future research, given the inconsistent results across samples.

\section{Limitations}

Due to the correlational nature of this study, causal relationships may not be determined. More research is needed to ascertain how and why these variables interact to produce these results, specifically those related to the proposed logarithmic structure and the predictors identified by the regression analysis. As noted earlier in the discussion, a longitudinal study is needed to determine how the relationships among these variables change over time.

Limitations with the measures include three points. Firstly, this study obtained self-reported height and weight measures that may be skewed by gender, current weight, and age. For example, in a study of 6,101 individuals from the National Health and Nutrition Examination Epidemiological Follow-Up study, Kovalchik (2009) found that women under-report their weight by $0.82 \mathrm{~kg}$, men overreport by $2.27 \mathrm{~kg}$, and each unit increase in BMI corresponds to a $0.91 \mathrm{~kg}$ underestimation in current weight. Secondly, there may be a distinction between "ideal" and "acceptable" BMI that was not uncovered. However, because of the consistency in the participants' answers (i.e., no evidence of overall bimodal answers suggesting a difference between "ideal" and "acceptable"), this seems unlikely. Thirdly, the measure of relative size was limited at the upper end with the highest option being "five or more sizes from ideal" and could be improved upon by including higher sizing options to better discriminate individuals grouped into the higher obese categories.

\section{Conclusions}

This unique study examined the demographic and anthropometric variables as predictors of ideal BMI using cross-sectional data of 3,906 U.S. residents. There is a logarithmic relationship between current BMI and ideal BMI wherein participants with higher current BMIs had higher and more varied ideal BMIs. Those at higher current BMI may have more varied ideal BMI because of the relative individual importance of both health concerns and satisfaction with body image. In particular, current BMI is a strong predictor of ideal BMI, more so for men than women. In support of the hypothesis, there are demographic and anthropomorphic predictors of ideal BMI, which include gender, relative size, and current BMI.

These findings can aid researchers and practitioners to better understand body image issues and confront epidemic levels of obesity-related crises, such as diabetes and cardiovascular pathologies. Because there appears to be a spectrum of satisfying weights rather than an absolute ideal across individuals, body image researchers may be able to use these findings to create more accu- rate measures of body image dissatisfaction. Including items that reflect these variables, particularly gender and current BMI, may enable professionals to discern which individuals have abnormal body image goals relative to others with similar demographic and anthropometric characteristics. Furthermore, like findings indicating that internalization of media images varies across individuals (see Yamamiya et al., 2005), these findings provide evidence that typical men and women do not report their ideals according to the media images they view. Knowledge that those at higher weights have greater variance in ideal BMI will be of particular interest to practitioners, as it furthers understanding how individuals at greatest health risk set personal goals. For example, an obese individual with prediabetes may want to lose weight but still have an overweight ideal BMI that will not be helpful enough to reduce symptoms of prediabetes. Physicians may use the finding that men are less likely than women to have a healthy ideal BMI and ideal clothing size to open conversations with male patients about truly healthy goals. Educators designing wellness programs for children and adolescents may also use these findings to reinforce healthy goals and resist the normative influence of a culture struggling with an obesity epidemic. Diversity in ideal BMI has implications for understanding weight loss motivations and strategies and merits further research.

\section{Acknowledgements}

SlimFast ${ }^{\mathrm{TM}}$ sponsored this research. These funds allowed for the national, cross-sectional pool of respondents, provided by Yankelovich.

\section{References}

Bessenoff, G. R., \& Snow, D. (2006). Absorbing society’s influence: Body image selfdiscrepancy and internalized shame. Sex Roles, 54, 727-731.

Bordo, S. (2003). Unbearable weight: Feminism, Western culture, and the body (10th ed.). Berkeley, CA: University of California Press.

Cash, T. F., Grant, J. R., Shovlin, J. M., \& Lewis, R. J. (1992). Are inaccuracies in self-reported weight motivated distortions? Perceptual and Motor Skills, 74, 209-210.

Crawford, D., Jeffery, R. W., \& French, S. A. (2000). Can anyone successfully control their weight? Findings of a three-year community-based study of men and women. International Journal of Obesity and Related Metabolic Disorders, 24, 1107-1110.

Fallon, A., \& Rozin, P. (1985). Sex differences in perceptions of desirable body shape. Journal of Abnormal Psychology, 94, 102-105.

Flegal, K. M., Carroll, M. D., Ogden, C. L., \& Johnson, C. L. (2002). Prevalence and trends in obesity among U.S. adults, 1999-2000. Journal of the American Medical Association, 288, 1723-1727.

Flegal, K. M., Carroll, M. D., Ogden, C. L., \& Curtin, L. R. (2010). Prevalence and trends in obesity among U.S. adults, 1999-2008. Journal of the American Medical Association, 303, 235-241.

Feingold, A., \& Mazzella, R. (1998). Gender differences in body image are increasing. Psychological Science, 9, 190-195.

Franzoi, S. L., \& Shields, S. A. (1984). The Body Esteem Scale: Multidimensional structure and sex differences in a college population. Journal of Personality Assessment, $48,173-178$.

Frederick, D. A., Fessler, D. M. T., \& Haselton, M. G. (2005). Do representations of male muscularity differ in men's and women's magazines? Body Image, 2, 81-86.

Frederick, D. A., Peplau, L. A., \& Lever, J. (2006). The swimsuit issue: Correlates of body image in a sample of 52,677 heterosexual adults. Body Image, 3, 413-419.

Fox, C. S., Massaro, J. M., Hoffman, U., Pou, K. M., Maurovich-Horvat, P., Liu, C. L., et al. (2007). Abdominal, visceral, and subcutaneous adipose tissue compartments: Association with metabolic risk factors in the Framingham Heart Study. Circulation, 116, 39-48.

Garrow, J. S., \& Webster, J. D. (1985). Quetelets's index (W/H sq) as a measure of fatness. International Journal of Obesity, 9, 147-153.

Grabe, S., Ward, M. W., \& Hyde, J. S. (2008). The role of media in body image concerns among women: A meta-analysis of experimental and correlational studies. Psychological Bulletin, 134, 460-476.

Gregory, C. O., Blanck, H. M., Gillespie, C., Maynard, L. M., \& Serdula, M. K. (2008). Health perceptions and demographic characteristics associated with underassessment of body weight. Obesity, 16, 979-989.

Grogan, S., Evans, R., Wright, S., \& Hunter, G. (2006). Experiences of anabolic steroid use; interviews with men and women steroid users. Journal of Health Psychology, $11,849-860$. 
Hassan, M. K., Joshi, A. V., Madhavan, S. S., \& Amonkar, M. M. (2003). Obesity and health-related quality of life: A cross-sectional analysis of the U.S. population. International Journal of Obesity, 27, 1227-1232.

Heatherton, T. F., Mahamedi, F., Striepe, M., Field, A. E., \& Keel, P. (1997). A 10 year longitudinal study of body weight, dieting, and eating disorder symptoms. Journal of Abnormal Psychology, 106, 117-125.

Heinberg, L. J. (1996). Theories of body image: Perceptural, developmental, and sociocultural factors. In J. K. Thompson (Ed.), Body image, eating disorders, and obesity: An integrative guide for assessment and treatment (pp. 27-48). Washington, DC: American Psychological Association.

Janssen, I., Heymsfield, S. B., Allison, D. B., Kolter, D. P., \& Ross, R. (2002). Body Mass Index and waist circumference independently contribute to the prediction of nonabdominal, abdominal subcutaneous, and visceral fat. American Journal of Clinical Nutrition, 75, 683-688.

Kaminski, P. L., \& Hayslip, B. (2006). Gender differences in body esteem among older adults. Journal of Women and Ageing, 18, 19-35.

Kashubeck-West, S., Mintz, L. B., \& Weigold, I. (2005). Separating the effects of gender and weight-loss desire on body satisfaction and disordered eating behavior. Sex Roles, 53, 505-518.

Keel, P. K., Baxter, M. G., Heatherton, T. F., \& Joiner, T. E. (2007). A 20-year longitudinal study of body weight, dieting, and eating disorder symptoms. Journal of Abnormal Psychology, 116, 422-432.

Kelley, C. C., Neufeld, J. M., \& Musher-Eizenman, D. R. (2010). Drive for thinness and drive for muscularity: Opposite ends of the continuum or separate constructs? Body Image, 7, 74-77.

Kovalchik, S. (2009). Validity of adult lifetime self-reported body weight. Public Health Nutrition, 12, 1072-1077.

Kuczmarski, R. J. (1992). Prevalence of overweight and weight gain in the United States. American Journal of Clinical Nutrition, 55, 495S-502S.

Leit, R. A., Pope, H. G., \& Gray, J. J. (2001). Cultural expectations of muscularity in men: The evolution of Playgirl centerfolds. International Journal of Eating Disorders, 29 90-93.

Levine, M. P., \& Piran, N. (2004). The role of body image in the prevention of eating disorders. Body Image, 1, 57-70.

Martz, D., Curtin, L., Williams, C., Burgess, H., \& Maphis, L. (2009, March). Gender and the psychology of clothing size. In Poster presented at the annual meeting of the Society for Personality and Social Psychology Tampa, FL.

Martz, D. M., \& The Segmentation Company. (2007). Psychology of size survey. Unpublished raw data.

Mazur, A. (1986). U.S. trends in feminine beauty and overadaptation. Journal of Sex Research, 22, 281-303.

McAdams, M. A., Van Dam, R. M., \& Hu, F. B. (2007). Comparison of self-reported and measured Body Mass Index as correlates of disease markers in U.S. adults. Obesity, 15, 188-196.

McCreary, D. R. (2002). Gender and age differences in the relationship between body mass index and perceived weight: Exploring the paradox. International Journal of Men's Health, 1, 31-42.

McCreary, D. R., \& Sadava, S. W. (2001). Gender differences in relationships among perceived attractiveness, life satisfaction, and health in adults as a function of body mass index and perceived weight. Psychology of Men and Masculinity, 2, $108-116$.

Mei, Z., Grummer-Strawn, L. M., Pietrobelli, A., Goulding, A., Goran, M. I., \& Dietz, W. H. (2002). Validity of body mass index compared with other body-composition screening indexes for the assessment of body fatness in children and adolescents. American Journal of Clinical Nutrition, 75, 978-985.

Mokdad, A. H., Ford, E. S., Bowman, B. A., Dietz, W. H., Vinivor, F., Bales, V. S., et al (2003). Prevalence of obesity, diabetes, and obesity-related health risk factors, 2001. Journal of the American Medical Association, 289, 76-79.

Morry, M. M., \& Staska, S. L. (2001). Magazine exposure: Internalization, self-objectification, eating attitudes, and body satisfaction in male and female university students. Canadian Journal of Behavioural Science, 33, 269-279.

Munoz, D. J., Lai, M., Chen, E. Y., Mansour, M., Fischer, S., Roehrig, M., et al. (2007). Why patients seek bariatric surgery: A qualitative and quantitative analysis of patient motivation. Obesity Surgery, 17, 1487-1491.
National Institutes of Health. (1998). Clinical guidelines for the identification, evalua tion, and treatment of overweight and obesity in adults. Bethesda, MD: Author.

Neighbors, L. A., \& Sobal, J. (2007). Prevalence and magnitude of body weight and shape dissatisfaction among university students. Eating Behaviors, 8, 429-439.

Ogden, C. L., Carroll, M. D., Curtin, L. R., McDowel, M. A., Tabak, C. J., \& Flegal, K. M. (2006). Prevalence of overweight and obesity in the United States, 1999-2004. Journal of the American Medical Association, 295, 1549-1555.

Olivardia, R., Pope, H. G., Borowiecki, J. J., \& Cohane, G. H. (2004). Biceps and body image: The relationship between muscularity and self-esteem, depression, and eating disorder symptoms. Psychology of Men and Masculinity, 5, 112-120.

Owens, T. E., Allen, M. D., \& Spangler, D. L. (2010). An fMRI study of self-reflection about body image: Sex differences. Personality and Individual Differences, 48 , 849-854.

Pope, H. G., Philips, K. A., \& Olivardia, R. (2000). The Adonis Complex: The secret crisis of male body obsession. Sydney: The Free Press.

Putterman, E., \& Linden, W. (2004). Appearance versus health: Does the reason for dieting affect dieting behavior? Journal of Behavioral Medicine, 27, 185-204.

Reas, D. L., Masheb, R. M., \& Grilo, C. M. (2004). Appearance vs. health reasons for seeking treatment among obese patients with binge eating disorder. Obesity Research, 12, 758-760.

Reeves, M. J., \& Rafferty, A. P. (2005). Healthy lifestyle characteristics among adults in the United States, 2000. Archives of Internal Medicine, 165, 854-857.

Revicki, D. A., \& Israel, R. G. (1986). Relationship between body mass indices and measures of body adiposity. American Journal of Public Health, 76, 992-994

Rusticus, S. A., \& Hubley, A. M. (2006). Measurement invariance of the Multidimensional Body-Self Relations Questionnaire: Can we compare across age and gender? Sex Roles, 55, 827-842.

Sauer, A. (2010, September). Are your pants lying to you? An investigation. In Esquire style blog. Retrieved from. http://www.esquire.com/blogs/mens-fashion/pantssize-chart-090710

Serdula, M. K., Mokdad, A. H., Williamson, D. F., Galuska, D. A., Mendlein, J. M., \& Heath, G. W. (1999). Prevalence of attempting weight loss and strategies for controlling weight. Journal of the American Medical Association, 282, 1353-1358.

Shapiro, J. R., \& Anderson, D. A. (2003). The effects of restraint, gender, and body mass index on the accuracy of self-reported weight. International Journal of Eating Disorders, 34, 177-180.

Spitzer, B. L., Henderson, A. H., \& Zivian, M. T. (1999). Gender differences in population versus media body sizes: A comparison over four decades. Sex Roles, 40 545-565.

Stice, E., \& Shaw, H. (2004). Eating disorder prevention programs: A meta-analytic review. Psychological Bulletin, 130, 206-227.

Sypeck, M. F., Gray, J. J., \& Ahrens, A. H. (2004). No longer just a pretty face: Fashion magazines' descriptions of ideal female beauty from 1959 to 1999. Internationa Journal of Eating Disorders, 36, 342-347.

Thompson, J. K. \& Cafri, G. (Eds.). (2007). The muscular ideal: Psychological, social and medical perspectives. Washington, DC: American Psychological Association.

Thompson, J. K., \& Heinberg, L. J. (1999). The media's influence on body image disturbance and eating disorders: We've reviled them, now can we rehabilitate them? Journal of Social Issues, 55, 339-353.

Tiggemann, M., \& Stevens, C. (1999). Weight concern across the life-span: Relationship to self-esteem and feminist identity. International Journal of Eating Disorders, 26, 103-106.

Tunaley, J. R., Walsh, S., \& Nicolson, P. (1999). "I'm not bad for my age": The meaning of body size and eating in the lives of older women. Ageing and Society, 19 741-759.

U.S. Census Bureau. (2001). Year 2000 census. Retrieved from. http://www.census. gov/prod/2001pubs/c2kbr01-1.pdf

U.S. Census Bureau. (2009). Current population survey. Annual social and economic (ASEC) supplement. Retrieved from. http://www.census.gov/hhes/www/ cpstables/032010/hhinc/new06_000.htm

World Health Organization. (1998). Obesity: Preventing and managing the global epidemic. Geneva, Switzerland: Author.

Yamamiya, Y., Cash, T. F., Melnyk, S. E., Posavac, H. D., \& Posavac, S. S. (2005). Women's exposure to thin-and-beautiful media images: Body image effects of mediainternalization and impact-reduction interventions. Body Image, 2, 74-80. 